inhibition of miR-125b. Gene ontology revealed apoptosis regulation as the main activated pathway. Apoptotic genes included BAK1, BMF and BBC3, which are part of the BCL2 apoptosis pathway and predicted targets of miR-125b. Consistent with the sequencing results, qPCR confirmed that miR-125b knockdown upregulated these genes 24,48 and 72 hours after transfection $(n=12, p<0.01$ for each). BAK1 showed the strongest induction, that was also confirmed on the protein level by Western blot. Accordingly, miR-125b knockdown resulted in an increased apoptosis (at least 1.5-fold, $n=10, p<0.01$ ) compared to scrambled controls, measured by Caspase-Glo $3 / 7$ assay 24,48 or 72 hours post-transfection. Consistently, miR-125b overexpression decreased apoptosis (by at least $50 \%$, $n=10, p<0.01$ ) at these time points. Cleaved caspase 3 was upregulated in antimiR-125b transfected cells (median 2.3 fold, $Q_{1,3} 1.6,4 ; n=10, p<0.01$ ) confirmed by Western Blot. Annexin $\mathrm{V}$ live assay showed prevailing of apoptosis after miR125b downregulation.

Conclusions: MiR-125b is downregulated in SSc skin and primary SSc dermal fibroblasts. MiR-125b downregulation increases apoptosis in dermal fibroblasts that might be a compensatory strategy against excessive fibrosis that could be used for therapeutic purposes.

Disclosure of Interest: A. Kozlova: None declared, E. Pachera: None declared, B. Maurer Grant/research support from: AbbVie, Protagen, EMDO, Novartis, German SSc Society, OPO foundation, congress support from Pfizer, Roche, Actelion. MSD. Patent licensed: mir-29 for the treatment of systemic sclerosis, A. Jüngel: None declared, J. Distler Shareholder of: 4D Science, Grant/research support from: Anamar, Active Biotech, Array Biopharma, BMS, Bayer Pharma, Boehringer Ingelheim, Celgene, GSK, Novartis, Sanofi-Aventis, UCB, Consultant for: Actelion, Active Biotech, Anamar, Bayer Pharma, Boehringer Ingelheim, Celgene, Galapagos, GSK, Inventiva, JB Therapeutics, Medac, Pfizer, RuiYi and UCB, G. Kania Grant/research support from: Bayer Pharma AG, Germany; conference support: Actelion, O. Distler Grant/research support from: Actelion, Bayer, Boehringer Ingelheim, Mitsubishi Tanabe Pharma and Roche; patent mir-29 for the treatment of systemic sclerosis licensed, Consultant for: Actelion, Bayer, Biogenldec, Boehringer Ingelheim, ChemomAb, espeRare foundation, Genentech/ Roche, GSK, Inventiva, Italfarmaco, Lilly, medac, Medlmmune, Mitsubishi Tanabe Pharma, Pharmacyclics, Novartis, Pfizer, Sanofi, Sinoxa and UCB DOI: 10.1136/annrheumdis-2018-eular.2309

\section{FRI0403 MITOCHONDRIAL DYSFUNCTION AND OXIDATIVE STRESS IN MYOSITIS: A CENTRAL PATHOGENIC PATHWAY FROM MOUSE TO MAN}

O. Boyer ${ }^{1}$, A. Meyer ${ }^{2}$, C. Boitard ${ }^{3}$, C. Abad ${ }^{1} .{ }^{1}$ Inserm U1234, Normandy University, Rouen; ${ }^{2}$ EA3072, Strasbourg University Hospital, Strasbourg; ${ }^{3}$ Inserm U1016, Cochin Institute, Paris, France

Background: Myositides are severe diseases leading to a bedridden state and possibly death. The lack of animal model with spontaneously-occurring autoimmune myositis has hampered pathophysiological and therapeutic research. Autoimmune-prone NOD mice represent an invaluable model of type 1 diabetes (T1D). Inducible T cell co-stimulator (ICOS) is involved in germinal centre reaction and induction of helper T cell responses. We developed a unique model of myositis by invalidating the ICOS pathway in NOD mice ${ }^{1}$. Muscle holoproteome analysis in diseased mice together with observations of mitochondrial dysfunction in patients with dermatomyositis ${ }^{2}$ suggest a main role of oxidative stress in disease pathogenesis.

Objectives: To determine the pathogenic role of oxidative stress and evaluate the effect of antioxidant therapy on $/ \cos ^{-/}$NOD myositis.

Methods: Disease course was studied in $/ \mathrm{COS}^{-/}$NOD mice by grip strength, locomotor analysis and MRI. Muscle pathology was evaluated after conventional stainings, or by immuno-enzymology or immuno-histochemistry. Muscle-infiltrating cells were characterised by flow cytometry. New autoantibodies were identified in mice by serum proteomic analysis and sought for in myositis patients by ALBIA. Mouse muscle proteomic and transcriptomic analyses were performed by LC-MS/MS (Orbitrap) and RT-PCR, respectively. Muscle free radical production was assessed by EPR. Mice were treated by prednisolone $(10 \mathrm{mg} / \mathrm{kg} / \mathrm{day})$ or $\mathrm{N}$ acetylcysteine $(2 \mathrm{~g} / \mathrm{L})$ in drinking water.

Results : $I \cos ^{-/-}$NOD mice did not develop diabetes. Instead, myositis spontaneously occurred with decreased grip strength, impaired cadence, reduced print area and death around 40 wks. Pathological muscle analysis revealed necrotic myofibers and important inflammatory infiltrates (CD4 ${ }^{+} \mathrm{T}$ cells, macrophages). Muscle lesions yielded MRI T2 hypersignals that regressed under steroids. CD4 ${ }^{+}$ $T$ cells transferred disease to NOD.scid recipients. Serum proteomic analysis revealed a new autoantibody directed against a mitochondrial antigen. It was found present in $\sim 1 \%$ individuals from a 700 myositis patient cohort. Oxydative stress was manifest in $I \cos ^{-/}$NOD muscle by augmented free radical production, $\mathrm{H}_{2} \mathrm{O}_{2}$ production-related atrophy, altered $\mathrm{O}_{2}$ consumption and dysregulation of several mitochondrial genes and proteins. $\mathrm{N}$-acetylcysteine partially prevented myositis or ameliorated established disease.
Conclusions: This work establishes $I \cos ^{-/-}$NOD mice as a unique paradigm of myositis. A new autoantibody was discovered. Oxidative stress is present in dis eased muscle. Antioxidant therapy is effective in a preventive or curative fashion. Together with our previous data in dermatomyositis patients ${ }^{2}$, this work indicates the central role of mitochondrial dysfunction and oxidative stress in myositis pathogenesis and opens new perspectives for therapy.

\section{REFERENCES:}

[1] Briet C, et al. The Spontaneous Autoimmune Neuromyopathy in ICOSL-/ NOD Mice Is CD4+ T-Cell and Interferon- $\gamma$ Dependent. Front Immunol. 2017;8:287.

[2] Meyer A, et al. IFN- $\beta$-induced reactive oxygen species and mitochondria damage contribute to muscle impairment and inflammation maintenance in dermatomyositis. Acta Neuropathol 2017;134:655.

Acknowledgements: This work was supported by the Association Française contre les Myopathies, the European Union and Normandie Regional Council. Europe gets involved in Normandie with European Regional Development Fund (ERDF).

Disclosure of Interest: O. Boyer Grant/research support from: CSL Behring Celogos, Consultant for: CSL Behring, Inova Diagnostics, A. Meyer: None declared, C. Boitard: None declared, C. Abad: None declared DOI: 10.1136/annrheumdis-2018-eular.1154

\section{FRI0404 CHARACTERISATION OF A MONOCYTES/ MACROPHAGES CELL SUBSET CO-EXPRESSING BOTH M1 AND M2 PHENOTYPE MARKERS IN SYSTEMIC} SCLEROSIS PATIENTS

S. Soldano ${ }^{1}$, A.C. Trombetta ${ }^{1}$, B. Ruaro ${ }^{1}$, V. Tomatis ${ }^{1}$, R. Brizzolara ${ }^{1}$ P. Montagna ${ }^{1}$, S. Paolino ${ }^{1}$, C. Pizzorni ${ }^{1}$, V. Smith ${ }^{2}$, P. Contini ${ }^{3}$, A. Sulli ${ }^{1}$, M. Cutolo ${ }^{1} .{ }^{1}$ Research Laboratory and Academic Division of Clinical Rheumatology, Department of Internal Medicine, University of Genoa, IRCCS Polyclinic San Martino Hospital, Genoa, Italy; ${ }^{2}$ Department of Rheumatology, Ghent University Hospital, Ghent, Belgium; ${ }^{3}$ Clinical Immunology, Department of Internal Medicine, University of Genoa, IRCCS Polyclinic San Martino Hospital, Genoa, Italy

Background: In the pathogenesis of systemic sclerosis (SSc), the immune cell activation is an important event that includes alteration in the macrophage polarisation. ${ }^{1}$

Macrophages may polarise into classically activated (M1), which are characterised by the expression of specific markers such as Toll-like receptors (TLR2 and 4) and costimulatory molecules (CD80 and CD86), or alternatively activated (M2), which are characterised by the expression of specific phenotype markers such as mannose receptor-1 (CD206) and macrophage scavenger receptors (CD204 and CD163). M2 are present in the circulation and in the skin infiltrate of SSc patients (pts), where they seem to contribute to fibrosis. ${ }^{2-4}$

Objectives: To characterise circulating M2 monocytes/macrophages from SSc pts and healthy subjects (HSs) by their co-expression of CD204, CD163 and CD206, as well as cells expressing both M1 and M2 phenotype markers.

Methods: Fifty-eight SSc pts (54 females/4 males, mean age $63 \pm 13$ years), fulfilling the new EULAR/ACR criteria for SSc, and 27 age-matched HSs were consec utively enrolled after Informed Consent was obtained. Peripheral blood was collected and the antibodies CD14-APC-Vio770 and CD45-VioGreen were used to identify the monocyte/macrophage lineage; CD204-VioBright-FITC, CD163 PE-Vio770 and CD206-PeerCP-Vio700 were used to characterise the M2 pheno type, whereas CD80-APC, CD86-VioBlue, TLR4-PE and TLR2-PE-Vio615 were used to characterise the M1 phenotype (Miltenij Biothech). Flow Cytometry analysis was performed using Navios Flow Cytometer and the related Navios analysis software (Beckman Coulter).

Results: In the $\mathrm{CD} 14^{+}$cell subset (monocytes), the $\mathrm{CD} 14^{+} \mathrm{CD} 163^{+} \mathrm{CD} 206^{+} \mathrm{CD} 204^{+}$cell percentage was significantly increased in SSc pts compared to HSs $(p=0.02)$. Inside the CD $14^{+} \mathrm{CD} 163^{+} \mathrm{CD} 206^{+} \mathrm{CD} 204^{+}$monocytes/macrophages a subset of cells co-expressing also TLR4, CD80 and CD86 was detected. This mixed population (M2/M1) of cells was significantly increased in SSc pts compared to HSs ( $\mathrm{p}=0.003)$.

At the same time, circulating monocytes/macrophages showing a full M2 phenotype and characterised as $\mathrm{CD} 204^{+} \mathrm{CD} 163^{+} \mathrm{CD} 206^{+}$cells were investigated independently of the expression of CD14, and they also resulted significantly increased in SSc pts compared to HSs $(p<0.0001)$.

In the CD204 ${ }^{+} \mathrm{CD} 163^{+} \mathrm{CD} 206^{+}$cell subset (M2), the percentage of cells express ing also TLR4, CD80 and CD86 (M1) was significantly increased in SSc pts com pared to HSs $(p<0.0001)$.

Conclusions: These results describe for the first time a subset of circulating cells belonging to the monocyte/macrophage lineage with a mixed phenotype, which are characterised by the expression of both M1 and M2 surface markers. These 